\title{
AN ADAPTIVE IMAGE SHARPENING SCHEME
}

\author{
Tzong-Jer Chen \\ School of Information Engineering, Baise University, Baise, Guangxi, 533000 China
}

\begin{abstract}
Image enhancement is one important process for digital images. This processing gives a better subjective visual appearance. The contours and texture are key features that contain important information essential to the visual quality aspect. Adaptive image enhancement schemes are proven better than global methods. An adaptive image sharpening method based on spatial statistics is proposed in this report to effectively sharpen image structures. A noisy Lena was pre-filtered using a low-pass filter to produce a blurred Lena. The residual image was obtained using average filtering and then subtracted from the original image. The high-pass residual image should be a combination of structure and noise. The autocorrelation of each pixel is calculated on the residual image and the image then is sharpened using a nimble filter based on the adaptive autocorrelation values. The PFOM results show that the similarity of adaptive sharpening is better than the global scheme. The proposed method will be further developed and applied to improve image acuity, de-noising and image quality improvement.
\end{abstract}

\section{KEYWORDS}

Image Sharpening, Spatial Statistics, Nimble Filter, PFOM

\section{INTRODUCTION}

Significant growth in digital image technology development in a wide range of fields has led to rapid expansions in applications. In many usage areas, digital image improvement technology behavior depends on the input image quality [Chen, T.J. et al, 2016.]. Image enhancement is one of the areas of digital image processing that involves altering the pixel intensity so that the processed image gives a subjectively better visual appearance. This is an essential pre-processing stage for various image processing systems. For different kinds of images, various image enhancement methods have been proposed. There is no unique algorithm that can provide the best visual output on a wide variety of input images [Neeraj K.S. and Arun K.S., 2017].

In digital images the key features are the contours, edges, and textures essential to the visual quality aspect [Fu, S. et al., 2007, Zohair A.A., 2018]. The visual appearance of an image may be improved by emphasizing its high frequency parts to enhance the edges and detail information. High frequency content enhancement, such as edge and detail information may significantly improve the visual appearance of an image [Polesel A. et al., 2000, Chen T.J. et al., 2017].

Image sharpening changes the visual quality without altering the content of the processed image. This is a computer vision and image processing scheme [Cao, G. et al., 2011]. Image sharpening can be defined as the improvement of apparent sharpness in an image that is supposed to be blurred [Haq N. et al., 2011]. Unsharp masking-based schemes have been widely used in increasing the sharpness of images. However, image sharpening is still an active research area due to the existence of various effective challenges, including the amplification of latent image noise [Sheppard A.P. et al., 2004], the introduction of overshoot [Kheradmand, A., \& Milanfar, P. (2015)], and over-sharpening effects [Zohair A.A., 2018].

A fast, efficient and simple filter for image sharpening sufficient for achieving satisfactory results was proposed. This work showed that the effect of this nimble filter is better than traditional Laplacian and unsharp mask filters [Zohair A.A., 2018.]. It was, nevertheless, a non-adaptive and global scheme. This may amplify the noise pixels as sharpened structures.

Adaptive schemes for image enhancement were recently proposed [Polesel A., et al., 2000, Fu S. et al., 2007, Gui Z.G. et al., 2012]. These algorithms enhance images while effectively decreasing the noise concurrently. The development of adaptive modules for image enhancement may be a good scheme [Chen 
T.J. et al, 2016.]. Recently, Chen et al. proposed using spatial statistics (SS) as a threshold for adaptive filtering and work well [Chen T.J. et al, 2016, 2017, Chen T.J. 2018, Lu R.H. and Chen T.J. 2018].

The SS calculation was initially direct on a noisy image. Categorizing the pixel belongs to noise or structure based on the SS calculation results. This method successfully accomplishes the dual objectives of amplifying the detailed areas and not increasing noise. [Chen T.J. et al, 2016, 2017]. Afterwards, the SS calculation was applied to a residual image [Chen T.J. 2018, Lu R.H. and Chen T.J. 2018]. A residual image is obtained using average filtering and then subtracted from the original image. The high-pass residual image should be a combination of boundary and noise. The SS is calculated on each pixel of the residual image, and the noisy image is then adaptively filtered using a low pass filter based on the SS values. The PSNR results in their report showed that image quality using adaptive filtering is significantly better than that obtained from global filtering [Chen T.J. 2018, Lu R.H. and Chen T.J. 2018].

In this paper SS is applied on a residual image and then sharpened using a nimble filter adaptively. Images were processed through the proposed sharpening approach followed by an edge map calculation using Canny's approach [Bao P., Zhang L. and Wu X., 2005., Neeraj K.S. and Arun K.S., 2017]. The edge map is also reported quantitatively in terms of Pratt's Figure of Merit (PFOM) [ Chabrier S. et al., 2006, Neeraj K.S. and Arun K.S., 2017]. The PFOM results show that the adaptive sharpening similarity is better than that obtained with the global scheme.

\section{METHODS AND MATERIALS}

A Lena image was used in this report to prove the assumption. Gaussian white noise was added to this image $(\mu=100, \sigma=10)$ first. The residual image may be obtained by subtracting the noisy Lena from the smoothed pre-filtered image ( $3 \times 3$ and $5 \times 5$ average filter) [Roychowdhury S. et al., 2015].

\subsection{The Spatial Statistics}

The SS named Moran statistics are used to evaluate the mapped data randomness by measuring the spatial autocorrelation [Cliff A.D .and Ord J.K., 1981]. This SS is a Z score of spatial correlation in a window. This $\mathrm{Z}$ value was proven to be a good index for determining image smoothness or sharpness [Chen T.J. et al, 2006, Shiao Y.H. et al, 2007]. This SS also showed that it has been shown to have ability to distinguish between image noise and edges [Chen T.J. et al, 2016]. Both Chen et al. and Hung et al. recently proposed adaptive filters using Moran statistics [Chen T.J. et al, 2016, 2017, 2018, Hung C.C. and Chang E.S., 2017]. Pixels with higher $\mathrm{Z}$ score are likely to be structured areas and lower $\mathrm{Z}$ is possible for noise. To improve image acuity, a pixel will perform sharpening if the $\mathrm{Z}$ score at this position is higher than a preset value. A pixel $\mathrm{Z}$ score lower than the preset is thought to be noise.

\subsection{A Sharpness Filter}

A new nimble filter was recently proposed to improve the sharpness of medical images utilizing specific spatial image information with a distinct tuning weight. This filter is described below:

$$
\mathrm{k}_{(\mathrm{x}, \mathrm{y})}=\left(\varphi \times \mathrm{f}_{(\mathrm{x}, \mathrm{y})}\right)+\left((1-\varphi) \times\left[\frac{\left(\mathrm{f}_{(\mathrm{x}, \mathrm{y}+1)}+\mathrm{f}_{(\mathrm{x}, \mathrm{y}-1)}+\mathrm{f}_{(\mathrm{x}+1, \mathrm{y})}+\mathrm{f}_{(\mathrm{x}-1, \mathrm{y})}\right)}{4}\right]\right)
$$

where $k_{(x, y)}$ is the resulting image; $x, y$ are spatial coordinates, $f_{(x, y)}$ is the original degraded image, $\varphi$ is a tuning weight that controls the amount of produced sharpness. It should fulfill $(\varphi>1)$, where higher values lead to shaper results. $f_{(x, y+1)}, f_{(x, y-1)}, f_{(x+1, y)}, f_{(x-1, y)}$ are horizontally and vertically shifted version of the original image [Zohair A.A., 2018].

\subsection{Pratt's Figure of Merit (PFOM)}

Pratt processes an empirical measure for two pixel sets comparison. This measure is one of the most commonly used. Given scaling factor $a(a=1 / 9)$, and separation distance of $d$, PFOM can be calculated as follows: [Chabrier S. et al., 2006, Neeraj K.S. and Arun K.S., 2017]

$\mathrm{PFOM}=\frac{1}{\mathrm{I}_{\mathrm{N}}} \sum_{\mathrm{i}=1}^{\mathrm{I}_{\mathrm{A}}} \frac{1}{1+\mathrm{a} \times \mathrm{d}^{2}}$ 
where $I_{N}=\max \left\{I_{I}, I_{A}\right\}, I_{I}$ and $I_{A}$ are the ideal and actual edge maps, respectively. Higher PFOM value indicates the similarity between the ideal edge map and the candidate map.

\section{RESULTS}

\subsection{SS on Residual Images}

The Moran $Z$ value of each pixel in the residual image was calculated with a $5 \times 5$ sliding window. A $Z$ histogram was produced by collecting the $Z$ values from all pixels. This $Z$ histogram has proven that the image noise and edges are individually in lower and higher areas [Chen T.J. et al, 2006, Shiao Y.H. et al, 2007]. The $Z$ histograms for two residual images from a noisy Lena was filtered using $3 \times 3$ and $5 \times 5$ average filters each, as noted in Figure 1. This figure shows that the histogram (winsz $=5$ ) is shifted to areas with higher $\mathrm{Z}$ values compared to winsz $=3$. This effect also indicated that a residual image from a bigger window $(5 \times 5)$ had higher structural information than a smaller one $(3 \times 3)$. The smaller window has contained more noise than the big one.

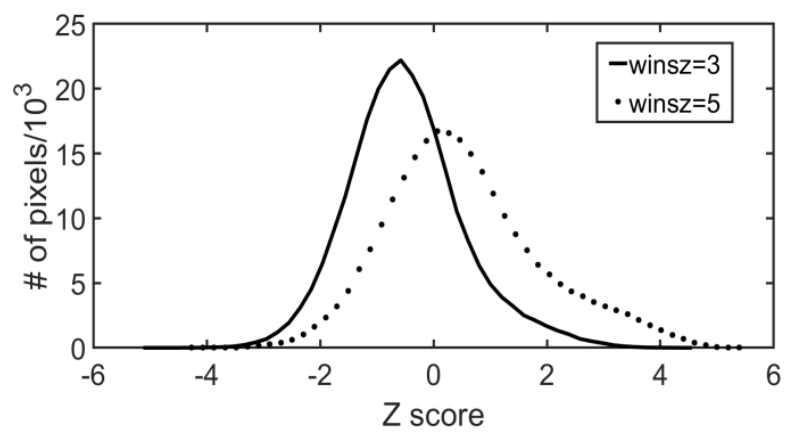

Figure 1. Histogram of $Z$ score for two residual images, ie. wins $Z=3(3 \times 3)$

\subsection{Adaptive Image Sharpening}

It is known that various medical devices introduce blur to recorded images due to many factual yet inevitable limitations. This nimble filter was originally applied to naturally blurred medical images [Zohair A.A., 2018].

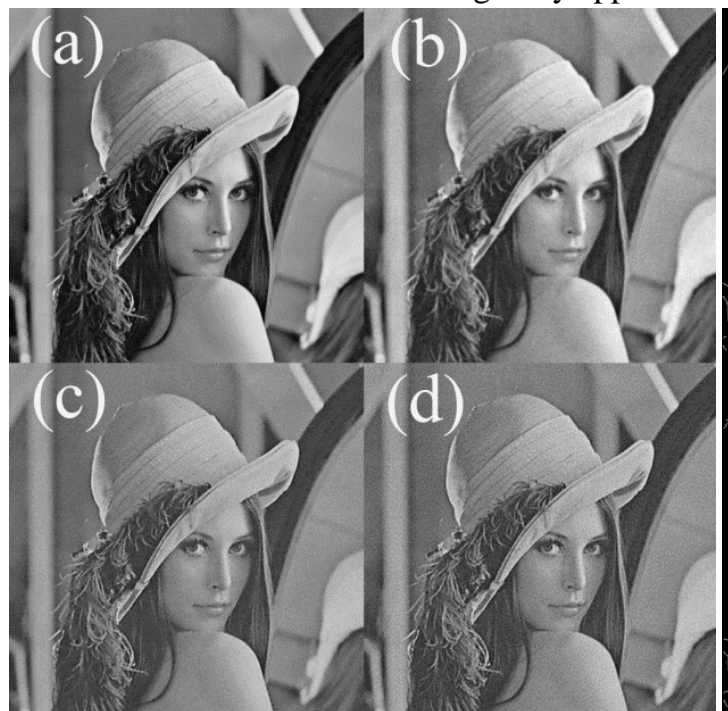

Figure 2. Lena images (a) original, (b) noisy, (c) Nimble filtered with $Z$ adaptive $(\varphi=6, Z>0)$, (d) Nimble filtered $(\varphi=6)$

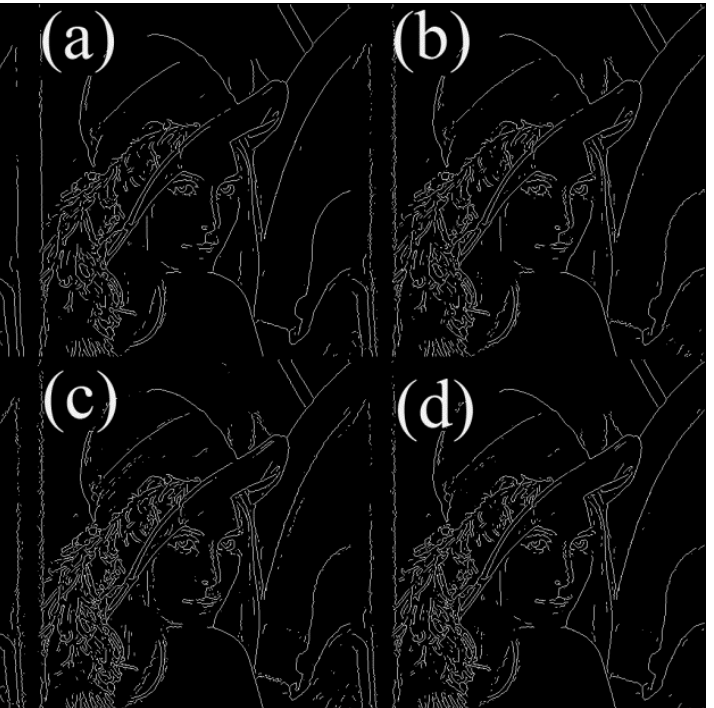

Figure 3. Edge map of Lena images (original), (b) noisy, (c) Nimble filtered with $Z$ adaptive $(\varphi=6, Z>0)$, (d) Nimble filtered $(\varphi=6)$ 
This new scheme was tested on a low-pass filtered noisy Lena image ( $3 \times 3$ average filter). The tuning weight $\varphi=6$ and 7 for nimble filter were used based on previous work [Zohair A.A., 2018]. Figure 2 shows the original Lena image, noisy $(\mu=100, \sigma=10)$, nimble filtered with $Z$ adaptive $(\varphi=6, Z>0)$, and Nimble filtered $(\varphi=6)$. Figure 3 shows an edge map of the images in Figure 2. These edge maps were obtained using Canny's approach with code support [Bao P., Zhang L. and Wu X., 2005.].

The PFOM calculates quantitative effects of sharpening scheme on the edge map, as noted in Table 1 . The higher PFOM indicates the similarity between the ideal edge (original Lena) and the edge map using the proposed scheme. The first row in Table 1 shows the PFOM values for the noisy Lena and those non-adaptive sharpening include $\varphi=6,7$. The $(3 \times 3)$ means the SS calculation was on a residual image produced using a winsz $=3$ average filter. Equally, the $(5 \times 5)$ means that the SS calculation was on a residual image produced using a winsz $=5$ average filter. The highest PFOM in this table is obviously at $\mathrm{Z}>0.0$ and $Z>1.0$ for $\varphi=6(3 \times 3)$ and for $\varphi=7(5 \times 5)$, individually. We found that the curve peaks are located around $\mathrm{Z}=0.0$ and 1.0 for winsz=3, 5 individually, as shown in Figure 1 . This effect indicates that sharpening on half of pixels may produce the best results.

Table 1. Quantitative performance (in terms of Pratt's Figure of Merit) PFOM

\begin{tabular}{lcc}
\hline \multicolumn{1}{c}{ Noisy Lena } & Nimble $\varphi=6(3 \times 3)$ & Nimble $\varphi=7(5 \times 5)$ \\
\hline Non-adaptive 0.895 & 0.9187 & 0.8950 \\
\hline Adaptive $(\mathrm{Z}>0.0)$ & $\mathbf{0 . 9 3 9 7}$ & 0.9142 \\
\hline Adaptive $(\mathrm{Z}>1.0)$ & 0.8416 & $\mathbf{0 . 9 3 8 7}$ \\
\hline Adaptive $(\mathrm{Z}>2.5)$ & 0.7612 & 0.8711 \\
\hline
\end{tabular}

\section{DISCUSSIONS}

This report proposes a new adaptive image sharpening scheme based on the results from spatial statistics. The SS Z score was applied to determine if an area belongs to the edge or noise before filtering. The PFOM calculation shows that the similarity between the edge maps is improved over the global image sharpening method. This scheme is effective for further image sharpening technology studies and improving image acuity.

The weight $(\varphi)$ and $\mathrm{Z}$ score turning on the variation of acuity and PFOM will be addressed in future works. This proposed scheme will also be applied to much more different images to verify the effectiveness. A noise model such as the Poisson distribution will be considered to simulate medical imaging systems and applied to medical images.

\section{ACKNOWLEDGEMENT}

This work was supported in part by grants from Baise University, Guangxi, China:

1. Construction project of characteristic specialty and experimental training teaching base (center) of Guangxi colleges and universities from 2018 to 2020: characteristic specialty of Electronic Information Engineering of Baise university (approval number: 52, Guijiao higher education [2018]; number: 119).

2. Project grant of master of engineering professional degree authorization point (electronic information) of School of Information Engineering, Baise University in 2019.

\section{REFERENCES}

Bao P., Zhang L. and Wu X., 2005. Canny edge detection enhancement by scale multiplication, IEEE. Trans. on Pattern Analysis and Machine Intelligence. 27, pp. 1485-1490.

Cao G. et al., 2011. Unsharp masking sharpening detection via overshoot artifacts analysis. IEEE Signal Processing Letters, 18 (10), pp. 603-606. 
Chabrier S. et al., 2006 .Supervised evaluation of synthetic and real contour segmentation results. European Signal Processing Conference (EUSIPCO) 2006, Florence, Italy.

Chen T.J. et al., 2006. A blurring index for medical images. J Digital Imaging, 19, pp. 118-125.

Chen T.J. et al., 2016. A pilot study on a new image enhancement method using spatial statistics. Joint International Information Technology, Mechanical and Electronic Engineering Conference (JIMEC 2016), Xian, China, pp. 33-38.

Chen T.J. et al., 2017. A NOVEL EDGE-PRESERVING FILTER FOR MEDICAL IMAGE ENHANCEMENT. Computer Graphics, Visualization, Computer Vision and Image Processing 2017, Lisbon, Portugal, pp. 279-384.

Chen T.J. et al., 2017. Compressed Medical Image Quality Determination Using the Kolmogorov Smirnov Test. Current Medical Imaging Reviews, 13 (2), pp. 204-209.

Chen T.J., 2018. MORAN'S Z BASED ADAPTIVE IMAGE FILTER. Computer Graphics, Visualization, Computer Vision and Image Processing 2018, Madrid, Spain. pp. 382-386.

Cliff A.D. and Ord J.K., 1981. Spatial process: models and applications. Pion, London, UK.

Fu S. et al., 2007. A feature-dependent fuzzy bi-directional flow for adaptive image sharpening. Neurocomputing, 70 (4), pp. 883-895.

Gui Z.G. et al., 2012. An X-ray image sharpening algorithm using nonlinear module. The Imaging Science Journal, 60: pp. 3-8.

Haq N. et al., 2011. Image sharpening by DWT-based hysteresis. In International conference on advanced concepts for intelligent vision systems . Ghent, Belgium, pp. 429-436.

Kheradmand A., \& Milanfar P., 2015. Non-linear structure-aware image sharpening with difference of smoothing operators. Frontiers in ICT, 2 (22), pp. 1-12.

Lu R.H. and Chen T.J., 2018. Adaptive Image De-noising Method Based on Spatial Autocorrelation. ISICDM 2018, Chengdu, China, pp. 125-128.

Neeraj K.S. and Arun K.S., 2017. An adaptive image sharpening scheme based on local intensity variations. SIViP, 11: pp. 777-784.

Polesel A. et al., 2000. Image Enhancement via Adaptive Unsharp Masking. IEEE Transactions on image processing, 9, pp. 505-510.

Roychowdhury S. et al., 2015. Blind analysis of CT image noise using residual denoised images. 2015 IEEE Nuclear Science Symposium and Medical Imaging Conference, San Diego, CA, USA.

Sheppard A. P. et al., 2004. Techniques for image enhancement and segmentation of tomographic images of porous materials. Physica A: StatisticalMechanics and its Applications, 339(1), pp. 145-151.

Shiao Y.H. et al., 2007. Quality of Compressed Medical Images. J of Digital Imaging, 20, pp.149-159.

Zohair A.A., 2018. Sharpness Improvement for Medical Images Using a New Nimble Filter. 3D Res, 9:12, pp. 2-12. 